\title{
A NEW EXAMPLE IN $K$-THEORY OF LOOPSPACES
}

\author{
TAHSIN GHAZAL
}

(Communicated by Frederick R. Cohen)

\begin{abstract}
The "Eilenberg-Moore" type spectral sequences which connect $K^{*}(\Omega X)$ and $K^{*}(X)$ have well-known bad properties, when, for example, $X=K(\mathbb{Z} / p, 2)$. This paper shows that the result can be as bad when $X$ is a finite complex.
\end{abstract}

The "Eilenberg-Moore" type spectral sequences which connect $K^{*}(\Omega X)$ and $K^{*}(X)$ have well-known bad properties, such as when $X=K(\mathbb{Z} / p, 2)$ [4]. The purpose of this note is to show that the spectral sequence properties can be just as bad when $X$ is a finite complex. Specifically, we prove

Theorem 1. For any odd prime $p$, there is a simply connected finite complex $X$ such that

(i) $\widetilde{K}^{*}(X ; \mathbb{Z})=0 \quad$ (integral $K$-theory)

(ii) $\widetilde{K}^{*}(\Omega X ; \mathbb{Z}) \quad$ contains a direct summand isomorphic to $\mathbb{Z} / p$.

It follows (informally) that there is no way of deducing $K^{*}(\Omega X ; \mathbb{Z})$ from the limit of the Eilenberg-Moore spectral sequence with $E_{2}=\operatorname{Tor}_{K^{*}(X ; \mathbb{Z})}(\mathbb{Z}, \mathbb{Z})$, even when $X$ is finite.

The example $X$ is a very obvious one; the cofibre of the Adams map [1] in a suitable dimension. We describe this space $X$ and prove that it has trivial $K$-theory in $\S 1$; in $\S 2$ we show that its loopspace has nontrivial $K$-theory.

\section{THE SPACE $X$}

Let $p>2$ be a prime and let $P^{n}(p)$ denote the Moore space $S^{n-1} U_{p} e^{n}$. In [1] J. F. Adams described stable maps

$$
A: P^{n+2 p-2}(p) \rightarrow P^{n}(p)
$$

( $n$ large) such that $A^{*}$ is an isomorphism in $K$-theory. We define $X(n, p)$ to be the cofibre of $A$ :

$$
X(n, p)=P^{n}(p) U_{A} C\left(P^{n+2 p-2}(p)\right) .
$$

Received by the editors August 25, 1988 and, in revised form, March 1, 1989.

1980 Mathematics Subject Classification (1985 Revision). Primary 55N15; Secondary 19L20.

$K e y$ words and phrases. Adams map, $K$-theory. 
Lemma 1. $\widetilde{K}^{*}(X(n, p) ; \mathbb{Z})=0$.

Proof. The proof follows immediately from the cofibration exact sequence.

Now choose $n$ so that $\widetilde{K}^{*}(\Omega X ; \mathbb{Z})$ is not 0 . The construction is only possible when $n$ has its minimum value $n_{0}$, since for $n>n_{0}, X$ is a suspension $\Sigma Y$, with $\widetilde{K}^{*}(Y ; \mathbb{Z})=0$. By the theory of the James construction [5], $\Omega X=\Omega \Sigma Y$ also has trivial $K$-theory in this case.

Cohen and Neisendorfer [3] have computed the minimum value for $n$ to be 3. We therefore set $X=X(3, p)=P^{3}(p) U_{A} C\left(P^{2 p+1}(p)\right)$. We have $H^{*}(X ; \mathbb{Z})$ given by

$$
\begin{aligned}
& H^{3}(X ; \mathbb{Z})=H^{2 p+2}(X ; \mathbb{Z})=\mathbb{Z} / p \\
& H^{j}(X ; \mathbb{Z})=0 \quad \text { otherwise. }
\end{aligned}
$$

\section{THE $K$-THEORY OF $\Omega X$}

Proving that the $K$-theory of $\Omega X$ is nontrivial is simple. Using (3) and the transgression exact sequence of [6] gives

Lemma 2. $H^{1}(\Omega X ; \mathbb{Z})=0 ; \quad H^{2}(\Omega X ; \mathbb{Z})=\mathbb{Z} / p$.

But $H^{2}(\Omega X ; \mathbb{Z})$ is a direct summand in $\widetilde{K}^{0}(\Omega X ; \mathbb{Z})$, as is well known. (The mapping $K(\mathbb{Z}, 2)=B U(1) \rightarrow B U$ is split by the determinant map.) This completes the proof of Theorem 1 .

We can prove the analogue to Theorem 1 for $K$-theory $\bmod p$ using the same space $X$; a careful use of the universal coefficient theorem [2] will provide a $\mathbb{Z} / p$ summand in $K^{0}(\Omega X ; \mathbb{Z} / p)$.

Of course the Adams maps are defined (in suitable degrees) if $p$ is replaced by $p^{r}$ :

$$
A_{r}: P^{n+2(p-1) p^{r-1}}\left(p^{r}\right) \rightarrow P^{n}\left(p^{r}\right) \text {. }
$$

If we know the exact level of desuspension of $A_{r}$, we can expect a similar result for $K$-theory of the cofibre and its loopspace.

\section{REFERENCES}

1. J. F. Adams, On the groups $J(X)$ IV, Topology 5 (1966), 21-71.

2. D. W. Anderson, Universal coefficient theorems for $K$-theory, Unpublished (1963).

3. F. R. Cohen and J. A. Neisendorfer, Note on desuspending the Adams map, Math. Proc. Cambridge Philos. Soc., 99 (1986), 59-64.

4. L. H. Hodgkin, The equivariant Künneth theorem in K-theory, Lecture Notes in Math., no. 496, Springer, Berlin 1975, pp. 1-101.

5. I. M. James, Reduced product spaces, Ann. of Math. 62 (2) (1955), 170-197.

6. J. P. Serre, Homologie singulière des espaces fibres, Ann. of Math. 54 (2) (1951), 425-505.

Mathematics Department, King Saud University, P.O. Box 2455, Riyadh 11451, Saudi ARABIA 